Claremont Colleges

Scholarship@ Claremont

All HMC Faculty Publications and Research

HMC Faculty Scholarship

$1-1-1994$

\title{
Resonance Raman Investigation of the Radical Cation of 1,3,5-Hexatriene
}

Tamas Keszthelyi

Riso National Laboratory

Robert Wilbrandt

Riso National Laboratory

Robert J. Cave

Harvey Mudd College

Jenifer L. Johnson

Harvey Mudd College

\section{Recommended Citation}

Keszthelyi, T.; Wilbrandt, R.; Cave, R.J.; Johnson, J.L.* “Resonance Raman Investigation of the Radical Cation of 1,3,5-Hexatriene,” J. Phys. Chem. 1994, 98, 5632. doi: 10.1021/j100073a010

This Article is brought to you for free and open access by the HMC Faculty Scholarship at Scholarship @ Claremont. It has been accepted for inclusion in All HMC Faculty Publications and Research by an authorized administrator of Scholarship @ Claremont. For more information, please contact scholarship@cuc.claremont.edu. 


\title{
Resonance Raman Investigation of the Radical Cation of 1,3,5-Hexatriene
}

\author{
Tamas Keszthelyi and Robert Wilbrandt \\ Department of Environmental Science and Technology, Riso National Laboratory, \\ DK-4000 Roskilde, Denmark
}

\author{
Robert J. Cave and Jenifer L. Johnson \\ Department of Chemistry, Harvey Mudd College, Claremont, California 91711 \\ Received: January 4, 1994; In Final Form: March 2, $1994^{\circ}$
}

\begin{abstract}
The resonance Raman spectrum of the 1,3,5-hexatriene radical cation generated by $\gamma$-irradiation in a Freon glass is reported. The spectrum is excited at $395 \mathrm{~nm}$ in resonance with the second absorption band. Identical spectra are obtained from ionized $(E)$ - and $(Z)-1,3,5$-hexatriene. The presence of more than one rotamer has to be assumed to account for all the observed resonance Raman bands. The bands are assigned to a mixture of the two rotamers, calculated at lowest energy, the $t t t$ and $t c t$ rotamers, on the basis of ab initio ROHF/6-31G calculated harmonic frequencies. The spectrum indicates that the $t t t$ and $t c t$ rotamers are formed in a ratio in the range $0.4 / 0.6-0.6 / 0.4$. Two possible mechanisms are proposed to explain the presence of these two rotamers.
\end{abstract}

\section{Introduction}

Polyene radical cations are species of general interest in the context of conductivity in conducting polymers, especially in polyacetylene. Upon doping, the conductivity of polyacetylene can be increased by several orders of magnitude.' Radical cations and anions correspond to positive and negative polarons in the terms of solid-state physics, and they are the primary species obtained upon doping of polyacetylene. The mechanism of photoconductivity in polyacetylene has been suggested to involve the formation of polarons, and recent experimental results seem to point toward the presence of polarons in photoexcited polyacetylene. $^{2}$

There has been extensive work investigating the spectroscopy and photochemistry of polyene radical cations. (For a recent review on radical ions see ref 3.) The radical cation of 1,3,5hexatriene, which is the subject of the present study, has been in the focus of numerous experimental as well as theoretical investigations. In the first experimental investigation the appearance energies of all important fragment ions were measured by electron impact mass spectrometry. ${ }^{4}$ Beez et al..$^{5}$ recorded the photoelectron (PE) spectrum of $(E)$ - and $(Z)$-1,3,5-hexatriene and interpreted them employing Koopmans' theorem using SPINDO orbital energies. Vibrationally resolved gas-phase emission spectra were reported by Allan and Maier, ${ }^{6}$ and the same group later carried out an investigation of radiative and fragmentation decay channels of hexatriene radical cations in their first electronic excited states by emission and photoelectronphotoion coincidence (PEPICO) spectroscopy. ${ }^{7}$ Laser photodissociation (PD) spectroscopy was employed in a study of the hexatriene radical cation by the groups of Dunbar ${ }^{8-10}$ and Morgenthaler and Eyler. ${ }^{11}$ While PE, ${ }^{5}$ PEPICO, ${ }^{7}$ and emission ${ }^{6,7}$ spectroscopies were able to distinguish between the radical cations derived from $(E)$ - and $(Z)$-hexatriene, identical PD spectra were obtained from both isomers. $8,9,11$ The group of Dunbar associated this spectrum with the $c i s$ isomer of the hexatriene radical cation, but later Bally et al..$^{12}$ suggested the PD spectrum to be that of the trans isomer. Several investigations on matrix-isolated hexatriene radical cations were also reported in the literature. Shida et al. ${ }^{13}$ carried out a detailed investigation of the lightinduced interconversions of the various rotamers of the hexatriene radical cation, as well as the photochemical ring opening of the

- Abstract published in Advance ACS Abstracts, April 15, 1994. cyclohexadiene radical cation in a glassy Freon matrix by electronic absorption (EA) spectroscopy. In this study four different rotamers could be identified from their EA spectra. The same problem was later reinvestigated by two groups 14,15 in $\mathrm{Ar}$ matrices. The experiments of Kelsall and Andrews ${ }^{14}$ confirmed the photochemical rearrangements but provided no new information for the identification of the intermediate rotamers. On the contrary, Bally et al.$^{15}$ presented evidence for five out of the six possible hexatriene rotamers and provided assignments for these. Laser-induced fluorescence excitation and resolved emission spectra of the radical cation of hexatriene were obtained in a $\mathrm{Ne}$ matrix be Bondybey et al. ${ }^{16}$ The spectra provided extensive vibrational information about the ground and first excited electronic states of the cation. In this study only one spectrum, presumably that of the trans-hexatriene radical cation, could be obtained ionizing any one of the $\mathrm{C}_{6} \mathrm{H}_{8}$ isomers.

As far as theoretical work is concerned, the PE spectrum of $(E)$ - and $(Z)$-1,3,5-hexatriene could successfully be explained in terms of SPINDO molecular orbital energies. ${ }^{5,17}$ Shida et al. ${ }^{13}$ used INDO and CNDO/S calculated potential energy surfaces to account for their experimental findings. In 1978, Dunbar and Teng 9 expressed concerns about the applicability of calculations neglecting configuration interaction for the description of excited electronic states of hexatriene radical cations. This view was strongly supported by the group of Bally, who successfully applied the semiempirical PPP-CI method to the interpretation of the electronic spectra of not only the hexatriene radical cation but also polyene radical cations in general. ${ }^{2,12}$ Schulz et al. ${ }^{18}$ employed the LNDO/S-PERTCI method to predict low-lying excited electronic states of the hexatriene radical cation. In the most recent theoretical work, by Cave and Johnson, ${ }^{19}$ ab initio ROHF and CASSCF calculations were performed on the ground and two low-lying excited electronic states of the hexatriene radical cation. In this work geometry optimization was carried out for all six possible rotamers, and vibrational analysis showed each of the optimized structures to be true minima. The ordering of the rotamers in the ground state was found to be $t t t, t c t, c t t, c t c$, $c c t$, and $c c c$, with energies of $0.0,2.70,3.37,6.90,8.32$, and 13.99 $\mathrm{kcal} / \mathrm{mol}$ relative to the most stable $t t$ rotamer at the CASSCF/ 6-31G level. The two lowest-energy rotamers thus correspond to the two stable isomers of the neutral molecule. CASSCF excitation energies to the two lowest-lying $\pi$ excited states were also obtained for each of the rotamers at the ground-state 
optimized geometries. These calculations supported the experimental assignments for the $t t t$ and $c c c$ rotamers 13,15 but indicated that an assignment of the remaining rotamers would be a difficult problem.

The spectroscopy and photochemistry of matrix-isolated radical ions is an active field with abundant documentation and several excellent review articles. ${ }^{3,20-22}$ The most frequently utilized methods for obtaining spectroscopic information about organic radical ions isolated in glassy matrices are $\mathrm{EA}^{3,20-23}$ and electron spin resonance (ESR) spectroscopy. 3,2122 Vibrational spectroscopy is a powerful tool to obtain structural information about minima on potential energy surfaces. Both infrared ${ }^{24-26}$ and resonance Raman ${ }^{27-30}$ spectroscopies have been applied to the studies of radical ions. In the present study, we apply the method of radiolytic generation of radical cations in a frozen Freon glass ${ }^{31}$ together with resonance Raman spectroscopy to characterize the hexatriene radical cation. Resonance Raman spectroscopy allows selective probing of the radical cations, which usually absorb at higher wavelength than their neutral precursors. ${ }^{32}$ It not only is a very useful tool to obtain structural information about the ground state but also can yield information about geometry changes occurring upon electronic excitation and about the dynamics on the resonant excited-state potential energy surface. ${ }^{33}$

\section{Methods}

(a) Theory. The theoretical vibrational frequencies were obtained from restricted open-shell Hartree-Fock (ROHF) results in the 6-31G basis. ${ }^{34}$ The calculations were performed using the program suite GAMESS. ${ }^{35}$ Due to the fact that the minima were located numerically to within a small but nonzero tolerance, the vibrational frequencies might change by a few reciprocal centimeters if tighter tolerances were used to converge the geometry. However, we expect this to have no real effect on the conclusions drawn here. As is common with Hartree-Fock frequencies, ${ }^{36}$ we expect that they are too large when compared with either the fundamentals or harmonic frequencies for the actual systems under study. For comparison purposes we have scaled all the calculated frequencies by a uniform factor of 0.9 . All quoted theoretical results are thus the scaled values.

One might wonder about whether the lack of polarization functions or the use of uncorrelated wave functions would have significant effects on the calculated vibrational frequencies. We have performed test calculations on ethylene and ethylene radical cation to assess this. We find that inclusion of polarization functions (via the $6-31 \mathrm{G}^{* *}$ basis $^{37}$ ) leads to some changes in the calculated frequencies, of on the order of $10-50 \mathrm{~cm}^{-1}$ for both the cation and the neutral. The changes in frequency in going from the unpolarized to polarized basis are not uniform in sign; some increase and some decrease. However, the order of states is maintained even with the changes in frequency, and within a given irreducible representation no significant changes in the spectrum occur. In addition, the alterations in frequency due to inclusion of polarization functions are modest relative to the effects due to the scaling discussed a bove. Since for the hexatriene radical cation we focus on only the totally symmetric modes, this suggests that whether or not polarization functions are included, similar conclusions would be drawn. Inclusion of correlation for ethylene at the second-order Møller-Plesset perturbation theory (MP2) level also has little effect. Of the 12 vibrational frequencies, MP2 switches the order of a $b_{2 g}$ and a $b_{1 \mathrm{u}}$ mode which are within $50 \mathrm{~cm}^{-1}$, but other than that the order of modes is maintained and the spectrum of totally symmetric modes is not altered significantly. We would note, however, that ethylene and ethylene radical cation do not possess very low-frequency modes (between 200 and $400 \mathrm{~cm}^{-1}$ ), and thus we cannot say with certainty that inclusion of correlation or polarization functions would not alter our results in this region. However, the assignments are largely made on the basis of the $400-1700-\mathrm{cm}^{-1}$ range, and thus the present tests lend support to the results for hexatriene. (b) Experiment. 1,3,5-Hexatriene was purchased from Aldrich as a mixture of isomers. This mixture, which is stabilized with $200 \mathrm{ppm}$ hydroquinone, contains $c a .66 \%$ of the $(E)$ - isomer. The separation of the isomers was carried out as described by Møller et al. ${ }^{38}$

$(Z)-1,3,5$-Hexatriene was isolated from the mixture via DielsAlder cycloaddition of the $(E)$-isomer to maleic anhydride. A $2.5-\mathrm{g}$ sample of the isomer mixture and $5 \mathrm{~g}$ of well-ground maleic anhydride were allowed to react for $2 \mathrm{~h}$ at room temperature while stirred and purged with dry $\mathrm{N}_{2}$ gas. The reaction mixture was then left standing overnight at $-20^{\circ} \mathrm{C}$. The $(Z)$-isomer was distilled off the reaction mixture in vacuum at room temperature and collected in a liquid nitrogen cooled receiver. The product was further purified by several freeze-pump-thaw cycles.

$(E)$-1,3,5-Hexatriene was prepared by iodine-catalyzed isomerization. Several crystals of sublimed iodine were added to $2.5 \mathrm{~g}$ of the isomer mixture. The reaction mixture was stirred and purged with dry $\mathrm{N}_{2}$ gas and allowed to react for $4 \mathrm{~h}$ at room temperature. The product $(E)$-isomer was then separated from the reaction mixture by transferring it to a liquid nitrogen cooled receiver on a vacuum line. With this procedure an isomeric purity of $93 \%$ could be achieved. The remaining $(Z)$-isomer was then removed on the vacuum line at $-20^{\circ} \mathrm{C}\left(\mathrm{CCl}_{4}\right.$ slush bath $)$. The product $(E)$-1,3,5-hexatriene was further purified by several freeze-pump-thaw cycles. For both $(E)$ - and $(Z)-1,3,5$ hexatriene the isomeric purity of the product was better than $95 \%$ as determined by gas chromatography (GC).

Capillary GC analysis was carried out on a J\&W DB1 column $(30 \mathrm{~m} \times 0.25 \mathrm{~mm})$ operated at $40^{\circ} \mathrm{C}$ with helium carrier gas in a Pye Unicam 104 chromatograph equipped with a flame ionization detector. Injector and detector temperatures of 200 ${ }^{\circ} \mathrm{C}$ were used. The chromatographic peaks were integrated on a Spectra Physics SP4290 integrator.

The solvents $\mathrm{CFCl}_{3}$ (Freon-11) and $\mathrm{BrCF}_{2} \mathrm{CF}_{2} \mathrm{Br}$ (Freon114B2) were purchased from Aldrich and used without further purification. Solutions $\left(5 \times 10^{-3} \mathrm{M}\right)$ of the hexatriene isomers in a $1: 1(\mathrm{v} / \mathrm{v})$ mixture of the above Freons were saturated with argon and frozen to form transparent glasses at $77 \mathrm{~K}$. The glasses were irradiated with doses of 0.05 and $0.2 \mathrm{Mrad}$ by ${ }^{60} \mathrm{Co} \gamma$-rays for measuring electronic absorption and resonance Raman spectra, respectively. The samples were then transferred to an Oxford Instruments DN1704 liquid nitrogen cryostat to perform the optical measurements. The samples were prepared in $7 \mathrm{~mm}$-i.d. cylindrical quartz cells for the resonance Raman measurements, while $7 \times 7 \mathrm{~mm}^{2}$ rectangular quartz cells were used to record electronic absorption spectra. For the resonance Raman measurements the cells were rotated at $8 \mathrm{rpm}$ in the cryostat.

Electronic absorption spectra were recorded on a Perkin-Elmer Lambda $5 \mathrm{UV} /$ vis spectrophotometer.

Resonance Raman spectra were excited in the second absorption band, in resonance with the strongly allowed ${ }^{2} \mathrm{~B}_{\mathrm{g}} \leftarrow{ }^{2} \mathrm{~A}_{\mathrm{u}}$ (in $C_{2 h}$ point group) electronic transition of the hexatriene radical cation. The 395-nm laser radiation of 1.0-2.0 mJ/pulse energy (15-ns pulses, $5-\mathrm{Hz}$ repetition rate) from an excimer (Lambda Physik EMG102E) pumped dye laser (Lambda Physik FL3002, Lambdachrome LC3690 laser dye) was focused on the bottom of the sample cell by a cylindrical lens. The intensity at the laser focus in the cell was approximately $1.0-2.5 \mathrm{MW} \mathrm{cm}^{-2}$. Scattered Raman light was collected at right angles and focused onto the entrance slit of a home-built $0.6-\mathrm{m}$ Czerny-Turner single monochromator (2400 grooves/mm holographic grating) through a GG420 Schott filter. A polarization scrambler was placed in front of the monochromator entrance slit. A slit width of $0.2 \mathrm{~mm}$ was used in all the experiments, resulting in a spectral resolution of $9 \mathrm{~cm}^{-1}$. Spectra were detected by a gated intensified optical multichannel analyzer (Spectroscopy Instruments OSMA IRY. 700 ) with 700 active channels. The calibration of the wavenumber scale was carried out using the Raman spectrum of indene as a 


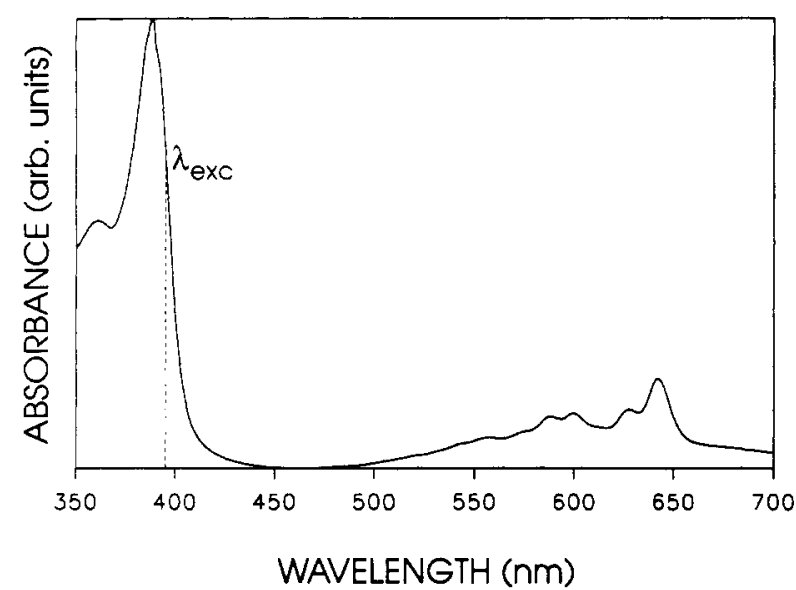

Figure 1. Electronic absorption spectrum of the cationic species obtained from $(E)$-1,3,5-hexatriene upon ionization by $\gamma$-irradiation in the Freon glass at $77 \mathrm{~K}$. An identical spectrum is obtained from $(Z)-1,3,5-$ hexatriene. The excitation wavelength for the resonance Raman spectrum of Figure $2 \mathrm{~A}$ is indicated as well.

reference.

Data handling was performed with a PDP11/23 computer. The 200-1800- $\mathrm{cm}^{-1}$ spectral range was covered combining three spectra, each covering $700 \mathrm{~cm}^{-1}$. A sample was usually exposed to 4000-6000 laser pulses. Increasing the number of laser pulses collected on one sample resulted in a significant decrease in intensity of the Raman bands of the cationic species, caused by local heating of the sample by the laser beam. Spectra of the hexatriene radical cation isomers in the Freon mixture (FM) at $77 \mathrm{~K}$ without $\gamma$-irradiation were also recorded and digitally subtracted from the spectra obtained from $\gamma$-irradiated samples applying empirically determined scaling factors. The spectra were corrected for the absorption of the GG420 filter.

Spectra were recorded with two different polarizations of the exciting laser beam to determine Raman depolarization ratios, using the method described by $\mathrm{Li}$ and Myers. ${ }^{39}$ To ensure pure polarization, a quartz Rochon polarizer was inserted into the laser beam. The polarization was turned by $90^{\circ}$ by a $\mathrm{MgF}_{2}$ Soleil-Babinet compensator. If the intensity of a Raman band recorded with the polarization of the exciting laser beam parallel to the optical axis of the monochromator is denoted by $I_{Y}$ and the intensity with the polarization of the exciting beam perpendicular to the optical axis of the monochromator by $I_{X}$, then the depolarization ratio $\rho$ is determined by the relation $\rho=I_{Y} /\left(2 I_{X}\right.$ $\left.-I_{Y}\right)$.

\section{Results}

All spectroscopic studies were carried out on $5 \times 10^{-3} \mathrm{M}$ argonsaturated solutions of hexatriene in the Freon mixture. The colorless glasses containing the $(E)$ - and $(Z)$-isomers of $1,3,5$ hexatriene turned deep blue as a result of $\gamma$-irradiation at liquid nitrogen temperature, indicating the formation of the hexatriene radical cation. The electronic absorption spectrum of the hexatriene radical cation in FM at $77 \mathrm{~K}$ is reproduced in Figure 1. In agreement with the observations of Shida et al., ${ }^{13}$ we obtained identical electronic absorption spectra following $\gamma$-irradiation of $(E)$ - and $(Z)-1,3,5$-hexatriene. This is in contrast with the results of Bally et al, ${ }^{15}$ who observed similar, but significantly different, spectra upon ionization of $(E)$ - and $(Z)$ 1,3,5-hexatriene in solid argon. Using the extinction coefficient of the hexatriene radical cation, $\epsilon=3 \times 10^{3} \mathrm{dm}^{3} \mathrm{~mol}^{-1} \mathrm{~cm}^{-1}$ at $642 \mathrm{~nm}$, determined by Shida et $a l .,^{13}$ it is estimated that about $15 \%$ of the hexatriene molecules were ionized at a dose of 0.2 Mrad of $\gamma$-irradiation.

The Raman spectra of $(E)$ - and $(Z)-1,3,5$-hexatriene solutions in $\mathrm{FM}$ at $77 \mathrm{~K}$ prior to $\gamma$-irradiation exhibited several strong bands due to the matrix material, as well as weak vibrational

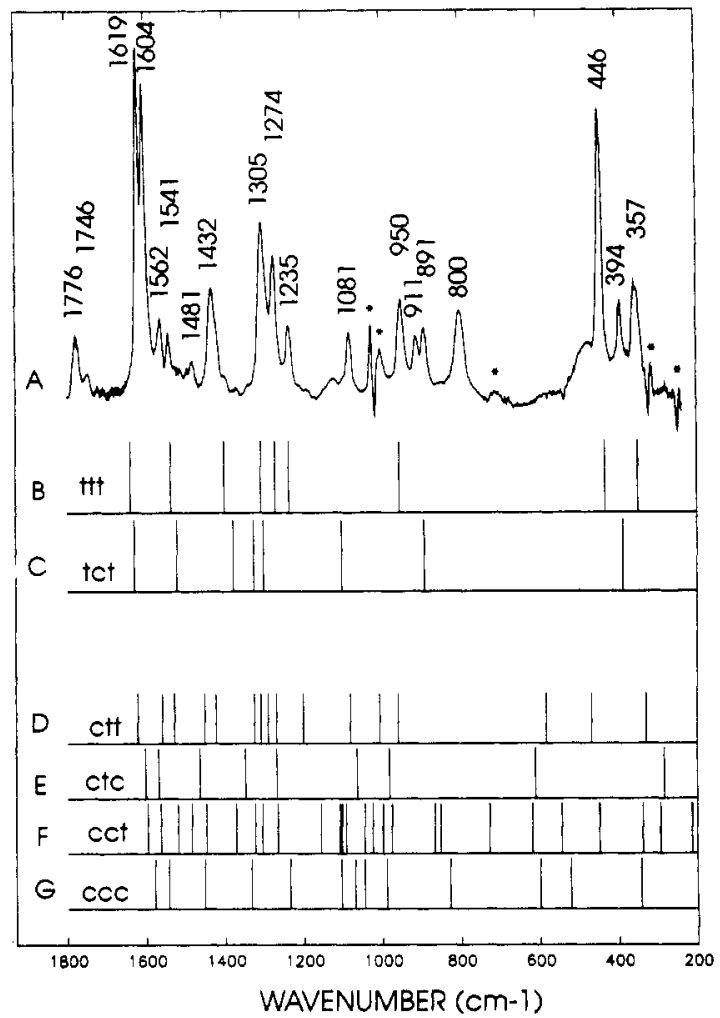

Figure 2. (A) Experimental resonance Raman spectrum of the cationic species obtained from $(E)$-1,3,5-hexatriene upon ionization by $\gamma$-irradiation in the Freon glass at $77 \mathrm{~K}$. An identical spectrum is obtained from $(Z)-1,3,5$-hexatriene. Solvent matrix and neutral hexatriene bands have been subtracted. The bands denoted by an asterisk are artifacts arising during the process of digital subtraction. (B)-(G) ROHF/6-31G calculated vibrational wavenumbers $\left(\mathrm{cm}^{-1}\right)$ of totally symmetric normal modes of the six stable rotamers of the hexatriene radical cation; the calculated wavenumbers were uniformly scaled down by a factor of 0.9 .

bands attributable to the ground state of the solute hexatriene isomers. Following $\gamma$-irradiation, 19 new bands appeared in the Raman spectra of samples of both 1,3,5-hexatriene isomers in the region $200-1800 \mathrm{~cm}^{-1}$, recorded with $395-\mathrm{nm}$ excitation. The difference spectrum, featuring the bands appearing upon $\gamma$-irradiation, is shown in Figure $2 \mathrm{~A}$. Although a slow decrease in intensity of these bands occurred during the measurements together with an increase in the matrix band intensities, no change in the relative intensities of the resonance Raman bands appearing upon $\gamma$-irradiation was observed. Similarly to the results of electronic absorption measurements, no difference was found between the resonance Raman spectra of the cationic species derived from $(E)$ - and $(Z)$-1,3,5-hexatriene. The depolarization ratio of all these Raman bands was also determined. The bands are all polarized, with a depolarization ratio $\rho=0.3-0.5$.

\section{Discussion}

Neutral hexatriene has two stable isomers, $E$ (trans) and $Z$ (cis), differing in their configuration around the central double bond. It has been established ${ }^{15,20}$ that in the hexatriene radical cation the distinction between single and double bonds, which can clearly be made in the neutral molecules, becomes meaningless. Cave and Johnson ${ }^{19}$ calculated the bond lengths of the $t t t$ rotamer as $\mathrm{C}_{1} \mathrm{C}_{2}=1.375, \mathrm{C}_{2} \mathrm{C}_{3}=1.408$, and $\mathrm{C}_{3} \mathrm{C}_{4}=1.399 \AA$ at the CASSCF/6-31G level. It is generally believed ${ }^{13-15,19}$ that there are six local minima on the potential energy hypersurface of the ground-state hexatriene radical cation, named rotamers, the geometries corresponding to these minima differing in their conformation around the three central carbon-carbon bonds. Thus, the six rotamers of the hextriene radical cation are called $t t t, t c t, c t t, c t c c c t$, and $c c c$, the letters indicating the configuration 


\section{SCHEME 1}

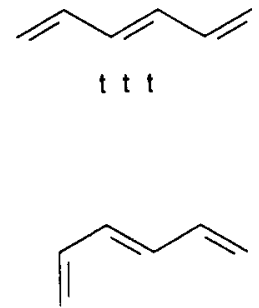

c t t

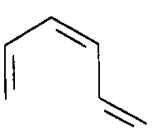

$\mathrm{cot}$

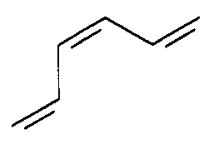

$\mathrm{t} c t$

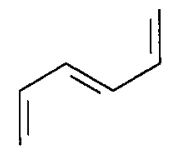

ct c

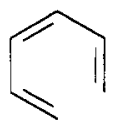

C C C (cis or trans) around the three central $\mathrm{CC}$ bonds. They are shown schematically in Scheme 1. The above ordering of the rotamers is according to their relative energies as determined by the calculations of Cave and Johnson, ${ }^{19}$ the most stable rotamer being the $t t t$.

If the Raman excitation wavelength is in resonance with a strong electronic transition, the resonance Raman spectrum is usually dominated by Franck-Condon scattering. In this case the vibrational modes observed with the strongest intensity are the totally symmetric ones. They gain intensity by changes in equilibrium geometry between the two resonant excited states and by changes in vibrational frequencies. It is usually the changes in geometry that give the dominant contribution. ${ }^{40}$ Hence, we attempt as assignment of the observed resonance Raman bands to totally symmetric vibrational normal modes. The normal modes having the greatest intensity then reflect the dominant changes in equilibrium geometry upon a transition between the two resonant electronic states.

First, we attempt to assign our observed resonance Raman spectra to only one rotamer of the hexatriene radical cation. Since experimental geometries for the rotamers of the hexatriene radical cation are not available, we base our analysis on the $a b$ initio results of Cave and Johnson. ${ }^{19}$ Their calculations yield $C_{2 \mathrm{~h}}$ symmetry for the $t t t$ and $c t c$ rotamers, $C_{2 v}$ for $t c t, C_{\mathrm{s}}$ for $c t t$, and $C_{2}$ for $c c c$, while the $c c t$ rotamer lacks any symmetry. All six rotamers have 36 normal modes of vibration. The vibrational normal modes of the $t t t$ and $c t c\left(C_{2 \mathrm{~h}}\right)$ rotamers divide into $13 \mathrm{a}_{\mathrm{g}}$ $+5 \mathrm{~b}_{\mathrm{g}}+6 \mathrm{a}_{\mathrm{u}}+12 \mathrm{~b}_{\mathrm{u}}$, those of the $t c t$ rotamer $\left(C_{2 v}\right)$ into $13 \mathrm{a}_{1}+$ $12 b_{1}+6 a_{2}+5 b_{2}$, those of the $c t t\left(C_{s}\right)$ into $25 a^{\prime}+11 a^{\prime \prime}$, and those of the $c c c$ rotamer $\left(C_{2}\right)$ into $19 \mathrm{a}+17 \mathrm{~b}$. The vibrational modes of the cct rotamer lack symmetry. With the exception of modes of ungerade symmetry, all modes may be Raman active. However, only fundamentals of totally symmetric modes or totally symmetric combinations of modes are expected to be resonance Raman active and polarized in the case of Franck-Condon scattering. The calculated wavenumbers of the totally symmetric modes for the six rotamers are shown schematically in Figure 2B-G.

Turning now to the experimental results, we observe 19 polarized bands in the $200-1800-\mathrm{cm}^{-1}$ region which we attribute to the hexatriene radical cation. This number is considerably higher than the number of totally symmetric modes of any of the $t t t(9), t c t(8)$, and $\operatorname{ctc}(9)$ rotamers in the region $200-1800 \mathrm{~cm}^{-1}$. Thus, none of the most stable rotamers can account for the observed bands alone. Neither can overtones, combination bands, or vibronic coupling account for unassigned bands under the assumption of the presence of only one rotamer.
One may speculate about the possible presence of dimer cations or other contaminations contributing to the observed resonance Raman spectrum. The electronic absorption spectra of radical cations of polyenic and aromatic systems often contain contributions from dimer cations. ${ }^{21,31,32,41}$ In fact, in the first electronic absorption spectra of butadiene, in $\gamma$-irradiated organic glasses ${ }^{42}$ most of the absorption bands were shown to derive from dimer cations by later workers. ${ }^{23,41}$ However, no observations of dimeric cations were reported in the case of hexatriene. ${ }^{13,23}$ The complications due to dimeric cation formation can usually be avoided by using sample concentrations lower than $10^{-2} \mathrm{M}^{23,31}$ At the concentration $\left(5 \times 10^{-3} \mathrm{M}\right)$ used in our experiments the interference from dimers seems unlikely. Moreover, the Raman spectra in this work have been recorded with the excitation wavelength in resonance with the strong second absorption band of the hexatriene radical cation. The electronic absorption spectrum in Figure 1 indicates no other species absorbing at this wavelength. Thus, vibrational Raman bands of the hexatriene radical cation are selectively enhanced, and we can confidently assign all the observed resonance Raman bands to the hexatriene radical cation.

This then leads us to the conclusion that at least two rotamers must contribute to the observed spectrum. We therefore in the following attempt the most simple approach, namely, an assignment of the observed resonance Raman spectrum to a combination of the two most stable, i.e., the $t t t$ and $t c t$, rotamers.

In the assignment of the resonance Raman spectrum, we make extensive use of the results of the theoretical calculations. As is seen below, the observed resonance Raman bands can be accounted for considering the energetically most stable $t t t$ and $t c t$ rotamers only. Nine bands can be readily assigned to fundamentals of the $t t t$ rotamer. These are the observed $357,446,950,1235,1274$, $1305,1432,1562$, and $1619 \mathrm{~cm}^{-1}$ bands, which are assigned to bands calculated at $350,433,955,1236,1271,1308,1400,1536$, and $1638 \mathrm{~cm}^{-1}$. Similarly, good agreement is found between the calculated vibrational wavenumbers of the $t c t$ rotamer and the wavenumber values of some as yet unassigned experimental bands. Thus, the observed $394,911,1081,1541$, and $1604 \mathrm{~cm}^{-1}$ bands are assigned to normal vibrations, calculated at $388,893,1102$, 1521 , and $1629 \mathrm{~cm}^{-1}$, of the $t c t$ rotamer of the hexatriene radical cation. The slightly asymmetric shape of the observed 1432$\mathrm{cm}^{-1}$ band, which we have already attributed to the $t t t$ rotamer, indicates that this band might actually consist of two overlapping bands. Consequently, we tentatively assign this band to normal vibrations of both the $t t t\left(1400 \mathrm{~cm}^{-1}\right)$ and the $t c t\left(1378 \mathrm{~cm}^{-1}\right)$ rotamers. This covers six of the eight calculated normal modes of the $t c t$ rotamer. The remaining two at 1302 and $1326 \mathrm{~cm}^{-1}$ either are probably hidden in the band triplet between 1235 and $1305 \mathrm{~cm}^{-1}$, which has already been assigned to the $t t t$ rotamer, or have very low intensity and are therefore not observed. This leaves only five experimental bands at $800,891,1481,1746$, and $1776 \mathrm{~cm}^{-1}$ unassigned. We assign these as overtone and combination bands of the $t t t$ and $t c t$ rotamers. The $800-\mathrm{cm}^{-1}$ band is broader than the other observed bands and therefore might also contain two overlapping bands. A likely assignment is to a combination band of the $357-$ and $446-\mathrm{cm}^{-1}$ modes of the $t t t$ and to the first overtone of the $394-\mathrm{cm}^{-1}$ mode of the $t c t$ rotamers. The $891-\mathrm{cm}^{-1}$ band is assigned to the first overtone of the $446-\mathrm{cm}^{-1} \mathrm{ttt}$ band. The $1746-$ and $1776-\mathrm{cm}^{-1}$ bands can tentatively be assigned as combination bands between 446 and $1305 \mathrm{~cm}^{-1}$, and 357 and $1432 \mathrm{~cm}^{-1}$ fundamentals of the $t t t$ rotamer, respectively. The $1481-\mathrm{cm}^{-1}$ band might arise as a combination band between the 394- and $1081-\mathrm{cm}^{-1}$ fundamentals of the $t c t$ rotamer. One of the characteristics of resonance Raman spectra is the presence of overtones and combinations of several modes. ${ }^{40}$ In the $200-1800 \mathrm{~cm}^{-1}$ wavenumber region investigated in the present work five bands (at $800,891,1481,1746$, and $1776 \mathrm{~cm}^{-1}$ ) are attributed to overtones or combination bands. Additional 
TABLE 1: Experimental Wavenumbers $\left(\mathrm{cm}^{-1}\right)$ of Resonance Raman Bands Attributed to Fundamentals, Overtones, and Combination Bands of the ttt and tct Rotamers of the 1,3,5-Hexatriene Radical Cation Together with ROHF/6-31G Calculated Wavenumbers* $\left(\mathrm{cm}^{-1}\right)$ of Fundamentals and Assignments of Overtone and Combination Bands

\begin{tabular}{rlllll}
\hline exptl & calcd $t t t$ & calcd $t c t$ & exptl & calcd $t t t$ & calcd $t c t$ \\
\hline & & 167 & 1274 & 1271 & 1302 \\
357 & 350 & & 1305 & 1308 & 1326 \\
394 & & 388 & 1432 & 1400 & 1378 \\
446 & 433 & & 1481 & & $\{394+1081\}$ \\
800 & $\{357+446\}$ & $\{2 \times 394\}$ & 1541 & & 1521 \\
891 & $\{2 \times 446\}$ & & 1562 & 1536 & \\
911 & & 893 & 1604 & & 1629 \\
950 & 955 & & 1619 & 1638 & \\
1081 & & 1102 & 1746 & $\{446+1305\}$ & \\
1235 & 1236 & & 1776 & $\{357+1432\}$ &
\end{tabular}

a All calculated wavenumbers were uniformly scaled down by a factor of 0.9 .

TABLE 2: Experimental and ROHF/6-31G Calculated Vibrational Wavenumbers $\left(\mathrm{cm}^{-1}\right)$ of Totally Symmetric Normal Modes $\left(\mathrm{a}_{\mathrm{g}}\right.$ in $C_{2 h}$ ) Together with Calculated Potential Energy Distributions (PED) for the $t t t$ Rotamer of the 1,3,5-Hexatriene Radical Cation

\begin{tabular}{rrl}
\hline exptl & calcd & \multicolumn{1}{c}{$\mathrm{PED}^{b}$} \\
\hline 1619 & 1638 & $\mathrm{rCH}(35), \nu \mathrm{C}_{3} \mathrm{C}_{4}(22), \nu \mathrm{C}_{2} \mathrm{C}_{3}(20), \nu \mathrm{C}_{1} \mathrm{C}_{2}(17)$ \\
1562 & 1536 & $\mathrm{sCH}_{2}(61), \nu \mathrm{C}_{1} \mathrm{C}_{2}(17), \nu \mathrm{CH}(10)$ \\
1432 & 1400 & $r \mathrm{CH}^{2}(59), \mathrm{sCH}_{2}(23), \nu \mathrm{C}_{1} \mathrm{C}_{2}(11)$ \\
1305 & 1308 & $\nu \mathrm{C}_{1} \mathrm{C}_{2}(39), \mathrm{rCH}(22), \mathrm{rCH}_{2}(15), \mathrm{sCH}_{2}(12)$ \\
1274 & 1271 & $\nu \mathrm{C}_{3} \mathrm{C}_{4}(45), \mathrm{rCH}(43)$ \\
1235 & 1236 & $\nu \mathrm{C}_{2} \mathrm{C}_{3}(32), \mathrm{dCCC}(23), \mathrm{rCH}(22), \mathrm{rCH}_{2}(11)$ \\
950 & 955 & $\mathrm{rCH}_{2}(64), \nu \mathrm{C}_{2} \mathrm{C}_{3}(13)$ \\
446 & 433 & $\mathrm{dCCC}^{2}(56), \nu \mathrm{C}_{2} \mathrm{C}_{3}(24), \nu \mathrm{C}_{3} \mathrm{C}_{4}(13)$ \\
357 & 350 & $\mathrm{dCCC}(89)$
\end{tabular}

all calculated wavenumbers were uniformly scaled down by a factor of $0.9 .{ }^{b}$ Only contributions greater than $10 \%$ are listed; $\nu=$ stretch, $\mathrm{s}=$ scissoring, $r=$ rock, and $d=$ deformation.

overtones or combinations of the remaining totally symmetric fundamentals may be active above $1800 \mathrm{~cm}^{-1}$, beyond the region of observation. The above assignment is listed in Table 1. The agreement between the calculated and observed vibrational wavenumbers is excellent, especially in the case of the $t t t$ rotamer. Additionally, the similar intensity of the strongest bands of the two rotamers (1619 and $1604 \mathrm{~cm}^{-1}$ ) suggests a ttt/tct ratio in the range $0.4 / 0.6-0.6 / 0.4$. We cannot, on the basis of the observed resonance Raman spectra, exclude the presence of other rotamers than $t t t$ and $t c t$. However, when comparing the observed and calculated spectra in Figure 2, it seems obvious that a superposition of calculated spectra of the $t t t$ and $t c t$ rotamers yields by far the best agreement between experiment and theory.

The nature of the normal modes is described by the potential energy distribution (PED), which gives the contribution of the internal coordinates to a particular normal mode. From the ROHF $/ 6-31 \mathrm{G}$ computed force field, we calculated the potential energy distributions of the normal modes. These are collected in Tables 2 and 3 for the $t t t$ and $t c t$ rotamers, respectively.

The most intense bands in the resonance Raman spectrum correspond to vibrational normal modes which reflect the dominant displacement of the excited state relative to the ground state. These are the normal modes calculated at 1638 and $433 \mathrm{~cm}^{-1}$ for the $t t t$ rotamer and the mode calculated at $1629 \mathrm{~cm}^{-1}$ for the $t c t$ rotamer. They are depicted in Figure 3, together with the 388$\mathrm{cm}^{-1}$ mode of the $t c t$ rotamer for comparison. On the basis of the observed resonance Raman spectrum, we conclude that the main geometric distortion of the excited state relative to the ground state of the radical cation is an in-plane distortion involving $\mathrm{CC}$ bond lengths and, in the case of the $t t t$ rotamer, $\mathrm{CCC}$ bond angles. All the observed resonance Raman bands could successfully be assigned to fundamentals of totally symmetric vibrational normal modes of the $t t t$ and $t c t$ rotamers and overtones and combinations
TABLE 3: Experimental and ROHF/6-31G Calculated Vibrational Wavenumbers ${ }^{2}\left(\mathrm{~cm}^{-1}\right)$ of Totally Symmetric Normal Modes $\left(a_{1}\right.$ in $C_{2 v}$ ) Together with Calculated Potential Energy Distributions (PED) for the tct Rotamer of the 1,3,5-Hexatriene Radical Cation

\begin{tabular}{rrl}
\hline exptl & calcd & \multicolumn{1}{c}{ PED $^{b}$} \\
\hline 1604 & 1629 & $\mathrm{rCH}(29), \nu \mathrm{C}_{1} \mathrm{C}_{2}(23), \nu \mathrm{C}_{3} \mathrm{C}_{4}(20), \nu \mathrm{C}_{2} \mathrm{C}_{3}(17)$ \\
1541 & 1521 & $\mathrm{sCH}_{2}(66), \nu \mathrm{C}_{3} \mathrm{C}_{4}(12), \nu \mathrm{C}_{1} \mathrm{C}_{2}(10)$ \\
1432 & 1378 & $\mathrm{rCH}(61), \nu \mathrm{C}_{3} \mathrm{C}_{4}(15)$ \\
1305 & 1326 & $\nu \mathrm{C}_{1} \mathrm{C}_{2}(43), \mathrm{sCH}_{2}(16), \mathrm{rCH}(15)$ \\
1274 & 1302 & $\mathrm{rCH}(60), \nu \mathrm{C}_{2} \mathrm{C}_{3}(22), \nu \mathrm{C}_{1} \mathrm{C}_{2}(10)$ \\
1081 & 1102 & $\mathrm{rCH}_{2}(40), \mathrm{rCH}^{2}(27), \mathrm{dCCC}(13), \nu \mathrm{C}_{3} \mathrm{C}_{4}(10)$ \\
911 & 893 & $\mathrm{rCH}_{2}(38), \nu \mathrm{C}_{2} \mathrm{C}_{3}(36), \nu \mathrm{C}_{3} \mathrm{C}_{4}(16)$ \\
394 & 388 & $\mathrm{dCCC}(72), \nu \mathrm{C}_{3} \mathrm{C}_{4}(12)$ \\
& 167 & $\mathrm{dCCC}(99)$
\end{tabular}

${ }^{a}$ All calculated wavenumbers were uniformly scaled down by a factor of $0.9{ }^{b}$ Only contributions greater than $10 \%$ are listed; $\nu=$ stretch, $s=$ scissoring, $r=$ rock, and $d=$ deformation.<smiles>CCC(C(C)C)C(CC)C(CC)C(C)C(C)C</smiles><smiles>CCC(=C(C(C)CC)C(CC)C(C)C)C(CC)C(C)C</smiles>

1629<smiles>CC(C)C(C)C(C)C(C)C(C)C(C)(C)C</smiles><smiles>CCC(C)C(C)C(C)C(C)C(C)C(C)C</smiles>

388
Figure 3. Selected normal modes of vibration of the $t t t$ and $t c t$ rotamers of the hexatriene radical cation.

of these modes. Thus, no observed bands are attributed to nontotally symmetric modes or their overtones. This apparent absence of overtones of out-of-plane modes seems to indicate that torsional distortion is unimportant in the electronic transition considered here.

Spectroscopic information about molecular vibrations of the $t t t$ and $t c t$ rotamers of the 1,3,5-hexatriene radical cation has previously been obtained by several experimental methods. Emission and photoelectron spectra ${ }^{7}$ yielded vibrational wavenumbers for both rotamers in the gas phase. For the $t t t$ rotamer the vibrationally resolved laser-induced fluorescence (LIF) spectrum was measured in a Ne matrix, ${ }^{16}$, and likely assignments of the vibrational bands were given. Nine fundamentals, $\nu_{5}-\nu_{13}$, and four overtone and combination bands were assigned, but several, mainly weak, bands were left unassigned. A hot band in the gas-phase photodissociation spectrum ${ }^{9}$ yielded one vibrational wavenumber for the $t c t$ rotamer, although later Bally $e t$ $a l .^{12}$ suggested reassignment of this spectrum to the $t t t$ rotamer. Our results also indicate the need for such reassignment. Vibrational wavenumbers from the above-mentioned references are compared to the values from the resonance Raman spectra measured in this work in Table 4. There is fair agreement between the results obtained by different spectroscopic methods and in different media. We note, however, about the assignments in ref 16 that in some cases the resonance Raman wavenumbers of bands we assign to the $t t t$ rotamer are in better agreement with wavenumbers of unassigned LIF bands than with those assigned to fundamental vibrations.

As has been demonstrated above, we could successfully assign the observed resonance Raman spectrum to a mixture of the two most stable, $t t t$ and $t c t$, rotamers of the hexatriene radical cation. We want to emphasize that the very same resonance Raman spectrum, as well as identical electronic absorption spectra, are obtained from both isomers of neutral hexatriene or from a mixture of the two isomers. Two alternative mechanisms are proposed for these observations. Both are in full agreement with all our 
TABLE 4: Comparison of the Experimental Vibrational Wavenumbers $\left(\mathrm{cm}^{-1}\right)$ of the $t$ tt and tct Rotamers of 1,3,5-Hexatriene Radical Cation in Their Ground Electronic State Obtained by Different Spectroscopic Methodsa

\begin{tabular}{ccccccccc}
\hline & \multicolumn{4}{c}{$t t$} & & \multicolumn{3}{c}{$t c t$} \\
\cline { 2 - 6 } \cline { 7 - 9 } mode & EM $^{b}$ & PES $^{b}$ & LIF $^{c}$ & RR $^{c}$ & PD $^{d}$ & EM $^{b}$ & PES $^{b}$ & RR $^{c}$ \\
\hline$\nu_{5}$ & 1560 & 1600 & 1622 & 1619 & & 1610 & & 1604 \\
$\nu_{6}$ & 1505 & & 1513 & 1562 & & 1500 & 1570 & 1541 \\
$\nu_{7}$ & 1380 & & 1376 & 1432 & & 1380 & & 1432 \\
$\nu_{8}$ & 1280 & 1300 & 1293 & 1305 & & & & 1305 \\
$\nu_{9}$ & 1230 & & 1239 & 1274 & & & $(1200)$ & 1274 \\
$\nu_{10}$ & & & 1115 & 1235 & & & & 1081 \\
$\nu_{11}$ & & & 951 & 950 & & & & 911 \\
$\nu_{12}$ & & & 442 & 446 & 350 & 390 & & 394 \\
$\nu_{13}$ & 345 & & 350 & 357 & & 170 & &
\end{tabular}

a EM stands for emission spectroscopy, PES for photoelectron spectroscopy, LIF for laser-induced fluorescence spectroscopy, PD for photodissociation spectroscopy, and RR for resonance Raman spectroscopy. ${ }^{b}$ Reference 7 , the $1200-\mathrm{cm}^{-1}$ vibration in the photoelectron spectrum of $(\boldsymbol{Z})-1,3,5$-hexatriene was considered questionable by the original authors. ${ }^{c}$ Reference $16 .{ }^{d}$ Reference $9 . e$ This work.

experimental results, and at present we are not able to choose between these possibilities. According to the first mechanism, which we consider more likely, an equilibrium mixture of rotamers is produced upon $\gamma$-irradiation, the dominating ones being the lowest-energy $t t t$ and $t c t$ rotmeric forms. The second mechanism, considered to be less likely, implies that the hexatriene radical cation is formed predominantly in the $t t t$ rotameric form upon $\gamma$-irradiation, and it is the exciting laser light which photolytically converts the $t t t$ rotamer to the $t c t$ and, possibly to a lesser extent, other rotameric forms. In the following we discuss these two mechanisms in some detail, with reference to previous experimental results.

In their paper, Shida et al. ${ }^{13}$ addressed the problem of identical electronic absorption spectra obtained upon ionization of the $(E)$ and $(Z)$-isomers of 1,3,5-hexatriene by $\gamma$-rays in FM at $77 \mathrm{~K}$. They attributed the observed spectrum to the $t t t$ rotamer. As an explanation, they suggested a mechanism supported by energetic considerations of the positive charge transfer between the Freon and hexatriene molecules, in which upon ionization of $(Z)$ hexatriene, the initially formed tct cation rotamer acquires sufficient excess energy to surmount the energy barrier separating it from the more stable $t t t$ rotamer. The same argument was invoked by Bondybey et al. ${ }^{16}$ to explain the identical LIF spectra in their Ne matrix experiments. On the contrary, Bally et al..$^{15}$ obtained different, but very similar, EA spectra from ionized $(E)$ - and $(Z)$-hexatriene. EA spectra obtained in polyatomic glasses usually show less resolution than in noble gas matrices. ${ }^{3}$ Therefore, it seems likely that electronic absorption spectra of the $t t t$ and $t c t$ rotamers in a Freon glass are very similar or identical. The spectrum assigned by Shida et al. ${ }^{13}$ to the $t t t$ rotamer may thus well arise from a mixture of the $t t t$ and $t c t$ rotamers. This seems even more likely in the light of the assignment of Bally et $a l .{ }^{15}$ who reassigned the EA spectrum that Shida et al. ${ }^{13}$ attributed to the $t c t$ rotamer to the $c t t$ rotamer. The first possible mechanism we propose is based on this assumption of identical EA spectra of the $t t t$ and $t c t$ rotamers. In this scheme we suggest that an equilibrium mixture of rotamers, dominated by the $t t t$ and $t c t$ rotamers, is formed during the ionization process from both $(E)$ and $(Z)-1,3,5$-hexatriene. This then results in the observation of identical EA and resonance Raman spectra of the ionized samples.

Adopting the original explanation of Shida et. al..$^{13}$ that the hexatriene radical cation formed during ionization in the Freon mixture is predominantly in the $t t t$ rotameric form leads to the second mechanism. To explain the presence of at least two rotamers, which is necessary to account for all the observed resonance Raman bands, we have to consider the nature of our experiment. The Raman spectra are excited at $395 \mathrm{~nm}$ in resonance with the strong second electronic absorption band of the $t t t$ rotamer of the hexatriene radical cation. According to Shida et al..$^{13}$ and Bally et al., ${ }^{15}$ the $t t t$ rotamer is efficiently converted into a mixture of all the rotamers upon illumination within this second absorption band. It is thus reasonable to assume that the $395-\mathrm{nm}$ exciting laser beam is inducing extensive rotamerization during the course of our experiment. Unfortunately, the relatively low signal-to-noise ratio of a single-pulse spectrum in our Raman measurements of samples in the Freon matrix does not allow positive identification of bands in spectra taken with less than 50-200 laser pulses. Spectra taken with these minimum numbers of pulses already show all 19 bands just like the spectra taken with the usual $1000-5000$ pulses. The $1.0-2.5 \mathrm{MW} \mathrm{cm}^{-2}$ intensity of the exciting laser beam may well be sufficient to establish a photostationary equilibrium between several rotamers with a small number of laser pulses, the $t t t$ and $t c t$ rotamers being the dominant ones.

\section{Summary and Conclusion}

In this work the resonance Raman spectrum of the 1,3,5hexatriene radical cation has been presented. The hexatriene radical cation was prepared by $\gamma$-irradiation in a glassy Freon matrix, and the resonance Raman spectrum was excited at 395 $\mathrm{nm}$ in resonance with the strongly allowed second absorption band. The spectra obtained from ionized $(E)$ - and $(Z)-1,3,5-$ hexatriene are identical but show more bands than could be accounted for if only one rotamer was present. The observed resonance Raman bands can, nevertheless, all be consistently assigned to a mixture of the two most stable, i.e., the $t t t$ and $t c t$, rotamers. The assignment is based on ab initio ROHF/6-31G calculated vibrational wavenumbers which give excellent agreement with the experimental values. The spectra indicate that under the present experimental conditions a mixture of the $t t t$ and $t c t$ rotamers is formed with a $t t t / t c t$ ratio in the range $0.4 /$ $0.6-0.6 / 0.4$. We propose two possible mechanisms to explain the presence of these two rotamers. It seems likely that $\gamma$-irradiation produces a mixture of rotamers, mainly the $t t t$ and $t c t$ from both neutral hexatriene isomers. Another possibility to be considered is that only the $t t t$ rotamer is produced upon ionization by $\gamma$-rays, and this rotamer is subsequently converted into an equilibrium mixture of rotamers, dominated by $t t t$ and $t c t$, upon laser irradiation during the initial stage of the Raman measurements.

We cannot establish with certainty which one of the above two schemes is responsible for the formation of the equilibrium mixture of the $t t t$ and $t c t$ rotamers. It is not known either whether the rotamerization takes place on the ground-state potential energy surface or whether excited states are also involved. Time-resolved resonance Raman experiments on the hexatriene radical cation generated by laser flash photolysis in the liquid phase are expected to reveal at least some of these details. Investigation of the radical cation of a "locked" hexatriene, in which isomerization is prevented by a bridging ring, may also help to get a deeper understanding of the elementary processes involved in the rotamerization of the hexatriene radical cation. Theoretical calculations on resonance Raman intensities of the different rotamers would assist their unambiguous identification. Vibrational spectra of isotopically substituted hexatriene radical cations would yield reliable force fields of the rotamers, which when compared with the force field of the neutral could characterize the changes in the chemical bonding occurring upon ionization.

Acknowledgment. We thank Prof. Thomas Bally (Fribourg) for fruitful discussions, Dr. Søren Møller (Roskilde University) for help with the separation of the hexatriene isomers and the gas chromatograph, and Drs. J. Fenger and K. B. Hansen and Mr. E. E. Larsen (Risø) for support with the experimental facility. T.K. gratefully acknowledges a grant from the Danish National Research Academy. This work was partially supported by the 
Danish Natural Science Research Council. R.J.C. acknowledges partial support of this work by a grant from the National Science Foundation and the Camille and Henry Dreyfus Foundation through the awarding of a 1993-1998 Camille and Henry Dreyfus Teacher Scholar award.

\section{References and Notes}

(1) Roth, S.; Bleier, H. Adv. Phys. 1987, 36, 385.

(2) Bally, T.; Roth, K.; Schrock, R. R.; Knoll, K.; Park, L. Y. J. Am. Chem. Soc. 1992, 114, 2440.

(3) Lund, A., Shiotani, M., Eds. Radical Ionic Systems, Properties in Condensed Phase; Kluwer Academic Publishers: Dordrecht, 1991

(4) Franklin, J. L.; Carroll, S. R. J. Am. Chem. Soc. 1969, 91, 6564.

(5) Beez, M.; Bieri, G.; Bock, H.; Heilbronner, E. Helv. Chim. Acta $1973,56,1028$

(6) Allan, M.; Maier, J. P. Chem. Phys, Lett. 1976, 43, 94.

(7) Allan, M.; Dannacher, J.; Maier, J.P. J. Chem. Phys. 1980, 73, 3114.

(8) Dunbar, R. C. J. Am. Chem. Soc. 1976, 98, 4671.

(9) Dunbar, R. C.; Teng, H. H. J. Am. Chem. Soc. 1978, 100, 2279.

(10) Hays, J. D.: Dunbar, R. C. J. Phys. Chem. 1979, 83, 3183

(11) Morgenthaler, L. N.; Eyler, J. R. J. Chem. Phys. 1981, 74, 4356.

(12) Bally, T.; Nitsche, S.; Roth, K.; Haselbach, E. J. Am. Chem. Soc. 1984, 106,3927 .

(13) Shida, T.; Kato, T.; Nosaka, Y. J. Phys. Chem. 1977, 81, 1095.

(14) Kelsall, B. J.; Andrews, L. J. Phys. Chem. 1984, 88, 2723.

(15) Bally, T.; Nitsche, S.; Roth, K.; Haselbach, E. J. Phys. Chem. 1985, 89,2528

(16) Bondybey, V. E.; English, J. H.; Miller, T. A. J. Mol. Spectrosc. $1980,80,200$.

(17) Bieri, G.; Burger, F.; Heilbronner, E.; Maier, J. P. Helv. Chim. Acto 1977, 60, 2213 467.

(19) Cave, R. J.; Johnson, J. L. J. Phys. Chem. 1992, 96, 5332.

(20) Haselbach, E.; Bally, T. Pure Appl. Chem. 1984, 56, 1203.

(21) Shida, T.; Haselbach, E. Bally, T. Acc. Chem. Res. 1984, 17, 180

(22) Shida, T. Annu. Rev. Phys. Chem. 1991, 42, 55.

(23) Shida, T. Electronic Absorption Spectra of Radical Ions (Physical Sciences Data 34); Elsevier: Amsterdam, 1988.
(24) Andrews, L. In Radical Ionic Systems, Properties in Condensed Phase; Lund, A., Shiotani, M., Eds.; Kluwer Academic Publishers: Dordrecht, 1991 ; $\mathrm{p} 55$.

(25) Szczepanski, J.; Roser, D.; Personette, W.; Eyring, M.; Pellow, R.; Vala, M. J. Phys. Chem. 1992, 96, 7876.

(26) Tang, W.; Zhang, X. L.; Bally, T. J. Phys. Chem. 1993, 97, 4373.

(27) Sheng, S. J.; Hug, G. Chem. Phys. Lett. 1978, 57, 168.

(28) Kato, T.; Muraki, N.; Shida, T. Chem. Phys. Lett. 1989, 164, 388.

(29) Kawashima, H.; Kato, T.; Shida, T. Chem. Phys. Lett. 1990, 165, 59.

(30) Kawashima, H.; Kato, T.; Shida, T. J. Raman Spectrosc. 1991, 22, 187.

(31) Iwamura, H.; Eaton, D. F. Pure Appl. Chem. 1991, 63, 1003.

(32) Bally, T. In Radical Ionic Systems, Properties in Condensed Phase; Lund A., Shiotani, M., Eds.; Kluwer Academic Publishers: Dordrecht, 1991 p 1.

(33) Myers, A. B.; Mathies, R. A. In Biological Applications of Raman Spectroscopy; Spiro, T. G., Ed.; John Wiley \& Sons: New York, 1987; Vol. $2, \mathrm{p} 1$.

(34) Hehre, W. J.; Ditchfield, R.; Pople, J. A. J. Chem. Phys. 1972, 56, 2257.

(35) GAMESS is a general purpose electronic structure program. The original program was assembled by M. Dupuis, D. Spangler, and J. J. Wendoloski at the National Resource for Computations in Chemistry, Software Catalog, University of California, Berkeley, CA (1980), Program OGO1. The current version is described in the Ouantum Chemistry Program Exchange Newsletter: Schmidt, M. W.; Baldridge, K. K.; Boatz, J. A.; Jensen, J. H.; Koseki, S.; Gordon, M. S.; Nguyen, K. A.; Windus, T. L.; Elbert, S. T. QCPE Bull. 1990, 10, 52

(36) Hehre, W. J.; Radom, L.; Schleyer, P. v. R.; Pople, J. A. Ab Initio Molecular Orbital Theory; John Wiley \& Sons: New York, 1986.

(37) Hariharan, P. C.; Pople, J. A. Theor. Chim. Acta 1973, 28, 213.

(38) Moller, S.; Langkilde, F. W.; Wilbrandt, R.J.Photochem. Photobiol. A: Chem. 1991, 62, 93 .

(39) Li, B.; Myers, A. B. J. Chem. Phys. 1988, 89, 6658.

(40) Siebrand, W.; Zgierski, M. Z. In Excited States; Lim, E. C., Ed.; Academic Press: New York, 1979; Vol. 4, p 1.

(41) Badger, B.; Brocklehurst, B. Trans. Faraday Soc. 1969, 65, 2576, $2582,2588$.

(42) Shida, T.; Hamill, H. J. Am. Chem. Soc. 1966, 88, 5371. 